Research Paper

\title{
Dietary glycine decreases both tumor volume and vascularization in a combined colorectal liver metastasis and chemotherapy model
}

Juste Maneikyte1,2, Augustinas Bausys ${ }^{1,2,3}$, Bettina Leber ${ }^{1}$, Angela Horvath ${ }^{4}$, Nicole Feldbacher ${ }^{1}$, Gerald Hoefler $^{5}$, Kestutis Strupas ${ }^{2}$, Philipp Stiegler ${ }^{1}$, Peter Schemmer ${ }^{1 凶}$

1. General, Visceral and Transplant Surgery, Dept. of Surgery, Medical University of Graz, Austria

2. Faculty of Medicine, Vilnius University, Lithuania

3. National Cancer Institute, Vilnius, Lithuania

4. Gastroenterology and Hepatology, Dept. of Internal Medicine, Medical University of Graz, Austria

5. Diagnostic and Research Institute of Pathology, Medical University of Graz, Austria

$\triangle$ Corresponding author: Univ.-Prof. Dr. med. Dr. h.c. Peter Schemmer, MBA, FACS, General, Visceral and Transplant Surgery, Dept. of Surgery, University Hospital Graz, Medical University of Graz, Auenbruggerplatz 29, A-8036 Graz, Austria. Phone: +43 31638584094 email: peter.schemmer@medunigraz.at

(c) Ivyspring International Publisher. This is an open access article distributed under the terms of the Creative Commons Attribution (CC BY-NC) license (https:// creativecommons.org/licenses/by-nc/4.0/). See http://ivyspring.com/terms for full terms and conditions.

Received: 2019.04.02; Accepted: 2019.05.02; Published: 2019.06.04

\begin{abstract}
Rationale: Chemotherapy (CTx) with FOLFOX is indicated prior to resection of liver metastases; however, its effect is limited due to chemoresistance and its toxicity prevents from aggressive surgery needed in some cases. Hepatoprotective glycine has been shown to have anti-tumorigenic properties in various cancers. Thus, this study was designed to evaluate the effects of glycine combined with FOLFOX on colorectal liver metastases (CRLM).

Methods: The effect of glycine combined with 5 -fluorouracil and oxaliplatin was investigated in vitro on colorectal cancer (CC531). Further, Wag/Rij rats with CRLM were treated with $5 \%$ dietary glycine \pm FOLFOX. $\mu$ CT liver scan, anti-Ki67, and anti-CD31 were compared.

Results: Glycine alone and combined with CTx has no effect on both CC531 viability in vitro and tumor proliferation in vivo; however, glycine significantly decreased tumor volume to about $42-35 \%$ of controls in vivo $(p<0.05)$ with a $60 \%$ decreased tumor microvascular density (MVD) $(p=0.004)$. Further glycine doesn't counteract anti-tumor properties of CTx.

Conclusions: This study nicely demonstrates that glycine inhibits the growth of CRLM and does not decrease CTx effectiveness. Underlying mechanisms most likely include a decreased tumor MVD. Clinical trials are warranted to implement non-toxic hepatoprotective glycine in novel anti-cancer strategies in humans.
\end{abstract}

Key words: Glycine, FOLFOX, colorectal liver metastasis

\section{Introduction}

Colorectal cancer (CRC) is the third most common malignancy and the fourth leading cause of cancer-related deaths worldwide (1-3). Surgery remains the only curative treatment option for this disease. However, metastases develop in every second CRC patient and the liver is the most common site (4) with resection being the only potential cure for this disease. Unfortunately, liver metastases are unresectable in up to $85 \%$ of patients initially $(4,5)$. In such cases, chemotherapy (CTx) is applied for resectability. FOLFOX (5-fluorouracil (5-FU), oxaliplatin (OX), leucovorin) is today's standard CTx for CRC and for its liver metastasis (CRLM). Also, various monoclonal antibodies against vascular endothelial growth factor (VEGF) or epidermal growth factor receptor can be combined together with the conventional CTx. Therefore, CTx is gaining importance in CRLM treatment, but a significant 
proportion of patients undergoing CTx still face treatment failure for chemoresistance $(6,7)$. Further CTx-toxicity leads to suboptimal treatment, impaired quality of life and increased medical costs (8). Thus, the development of novel more effective non-toxic CTx regiments is necessary to improve the survival of CRC patients.

Non-toxic amino acid glycine (9-12) has anti-tumorigenic effect i.e. in experimental melanoma and hepatocellular carcinoma $(13,14)$. Previous in vivo studies have demonstrated that glycine protects from FOLFOX- / FOLFIRI-induced liver injury (15). Thus, glycine may be an attractive anti-cancer agent for the treatment of CRLM; however, there is no in vivo data available on anti-cancer effects on CRLM of glycine in vivo. In contrast, there is limited data on the potential pro-tumorigenic effects of glycine on fast proliferating tumor cells $(12,13)$. Further, it is unclear whether glycine impairs the anti-tumorigenic potential of 5-FU and OX both included in FOLFOX (17).

Thus, this study was designed to evaluate the effects of glycine combined with FOLFOX on CRLM both in vivo and in vitro.

\section{Methods}

\section{Cell culture}

Cell line and viability testing. A rat colorectal cancer cell line (CC531) (Cell Lines Service, Eppelheim, Germany) was cultivated at $37^{\circ} \mathrm{C}, 5 \% \mathrm{CO}_{2}$ using RPMI-1640 medium supplemented with $10 \%$ fetal bovine serum (GE Healthcare Life Sciences, Utah, USA), $1 \%$ penicillin / streptomycin, 1\% L-glutamine and $25 \mathrm{mM}$ HEPES for the experiments. For viability testing, the 3-(4,5-dimethylthiazol-2-yl)5-diphenyltetrazolium bromide (MTT) assay was used exactly as described by the manufacturer. Briefly, ten microliters of MTT (Sigma Aldrich, St. Louis, MO, USA) solution were added to the cells and incubated for $2 \mathrm{~h}$ (CC531) at $37^{\circ} \mathrm{C}$. The medium was then discarded, and the precipitated formazan crystals were dissolved using dimethyl sulfoxide (Sigma Aldrich, St. Louis, MO, USA). Optical density was measured after 30 minutes at $570 \mathrm{~nm}$ using a Spectroxtar microplate reader (BMG labtech, Ortenberg, Germany). Cell viability was calculated as a percentage of vehicle control. All experiments were performed in duplicates and repeated three times.

Impact of glycine on CC531 cells. To determine the effects of glycine on cells, CC531 were cultivated for $24 \mathrm{~h}$ in 96-well plates under standard conditions in glycine-free media. For conditioning of cells, media was supplemented with various concentrations of glycine (Control (0), 0.05, 0.1, 0.25, 0.5, 1, 2, 4 and 8
$\mathrm{mM}$ ) (Carl Roth, Karlsruhe, Germany) and incubated for 48 hours. After incubation cell viability was measured using the MTT test as described above.

Glycine interaction with 5-fluorouracil and oxaliplatin. Glycine interaction with 5-FU and OX was tested on CC531 cells by exposure of CC531 cells to 5-FU and OX at the half maximal inhibitory concentration (IC50) combined with glycine at concentrations ranging from 0.1 to $5 \mathrm{mM}$. After $48-\mathrm{h}$ exposure cell viability was evaluated using MTT assay as described above.

The IC50 of each agent was calculated from the dose-response curves after CC531 cells were treated with 5-FU and $\mathrm{OX}$ at concentrations ranging from 1 to $800 \mu \mathrm{M}$ for $48-\mathrm{h}$. Cell viability was measured using the same MTT assay.

\section{Animal experiments}

All animal experiments were performed in accordance with the principles of laboratory animal care and national laws. The study protocol was approved by the Austrian ministry for science, research, and economy. Seven weeks old male WAG/Rij rats (weight 150 - $220 \mathrm{~g}$ ) were purchased from Charles River (Wilmington, MA, USA) and kept under standard laboratory conditions in the animal facility of the Medical University of Graz, Austria. A 12:12 h light: dark cycle was maintained. Prior to the experiments, rats were allowed to acclimatize to the new surroundings for 7 days. All animals had access to water and chow ad libitum. During the study, rats were inspected by animal caretakers on a daily basis and weighed 5 days before tumor implantation and on the days $0,7,10$ and 14 .

Experimental protocol. The study design is displayed in figure 1. A total of thirty-six WAG/Rij rats were divided into 2 groups. While 18 rats were fed with a glycine enriched diet (modified C1000 diet 15\% casein plus 5\% glycine; Altromin Spezialfutter, Lage, Germany), controls received an isonitrogenous balanced casein diet for 5 days (control diet: 20\% casein; Altromin Spezialfutter, Lage, Germany) before CRC liver metastasis was induced by injecting CC531 cells in $100 \mu \mathrm{l}$ of phosphate-buffered saline (PBS) into the right liver lobe as described below. On day 7 after tumor injection, the casein and glycine group were split into 2 subgroups treated with either FOLFOX or saline on the days 7 and 8 . CTx comprised on day 7 of two hundred $\mathrm{mg} / \mathrm{m} 2$ calcium folinate (Sandoz, Holzkirchen, Germany), $85 \mathrm{mg} / \mathrm{m} 2$ OX (Fresenius Kabi, Bad Homburg, Germany) and $1000 \mathrm{mg} / \mathrm{m} 2$ 5-FU (Sandoz, Holzkirchen, Germany) and was injected intraperitoneally. The second dose was given on day 8 comprising of $200 \mathrm{mg} / \mathrm{m} 2$ calcium folinate and $1000 \mathrm{mg} / \mathrm{m} 2$ 5-FU. The doses were calculated 
according to the animal skin surface as described elsewhere (15).

All study-related procedures were performed at approximately the same daytime to avoid differences caused by natural circadian rhythms.

Tumor implantation. For tumor implantation anesthesia was induced by isoflurane $(2 \%, 21 / \mathrm{min})$ and fentanyl $(5 \mu \mathrm{g} / \mathrm{kg})$ application. Animals were placed in a supine position on an automatically regulated heating pad; body temperature of $37^{\circ} \mathrm{C}$ was maintained through the procedure. Under continuous isoflurane $(2 \%, 21 / \mathrm{min})$ inhalation a right subcostal incision was performed. The right liver lobe was mobilized, and $100 \mu \mathrm{l}$ (5x106 cells) of cell suspension in PBS was injected under the liver capsule. The laparotomy was closed by two layers of single-node sutures (Vicryl 4-0, Ethicon, Somerville, NJ, USA). The skin was adapted and glued with tissue glue (Mayer-Haake, Ober-Mörlen, Germany). The procedure-related pain was managed with a single subcutaneous injection of carprofen $(4 \mathrm{mg} / \mathrm{kg})$ and by addition of ibuprofen $(0.4 \mathrm{mg} / \mathrm{ml})$ to the drinking water for 4-5 days.

Blood sample analysis. Venous blood samples were collected at day 0, 7, 10 and 14 from the subclavian vein under isoflurane anesthesia. Complete blood count (CBC) was measured using a V-Sight hematology analyzer (A. Menarini Pharma $\mathrm{GmbH}$, Vienna, Austria). Glycine concentration was measured in samples taken on day 14 in a routine hospital lab from blood samples collected on the last day of the study. $\mu C T$ examination. $\mu \mathrm{CT}$ examination (Siemens Inveon MultiModality, Knoxville, TN, USA) was performed under isoflurane $(2 \%, 21 / \mathrm{min})$ anesthesia on day 7 and 14. The scanning parameters were set to a voltage of $55 \mathrm{kV}$, a current of $500 \mu \mathrm{A}$, an exposure time per projection image of $600 \mathrm{~ms}$, full $360^{\circ}$ rotation with 360 projection steps, binning of $2 \times 2$ and a field of view $64 \mathrm{~mm} \times 64 \mathrm{~mm}$ (2-bed positions) according to standard protocols. Variable magnification settings of the scanner were set to a low level in order to reach an effective pixel size of $52 \mu \mathrm{m}$. A commercially available contrast agent for preclinical imaging (ExiTron nano 12000; Miltenyl Biotec, Auburn, CA, USA) was used to contrast the liver and the tumor. A single injection of $700 \mu \mathrm{l}$ of contrast agent into the tail vein was performed 24 hours prior to the first scan.

Image analysis of CT. Analysis of reconstructed pictures was performed using the Mimics research v.20 software (Materialise, Leuven, Belgium). A mask with values ranging from -602 to -247 Hounsfield Units (HU) was created for the region of interest (ROI). Mask splitting function was used to mark the liver and to get rid of non-liver tissue. A 3D model of the liver was calculated, and the volume was measured. Later a mask with values ranging from -696 to $-574 \mathrm{HU}$ was created for a liver area with the tumor. Mask splitting function was used to eliminate nontumor tissue. A 3D model of the tumor was calculated, and the volume was measured. Two independent reviewers performed measurements of the tumor volume.

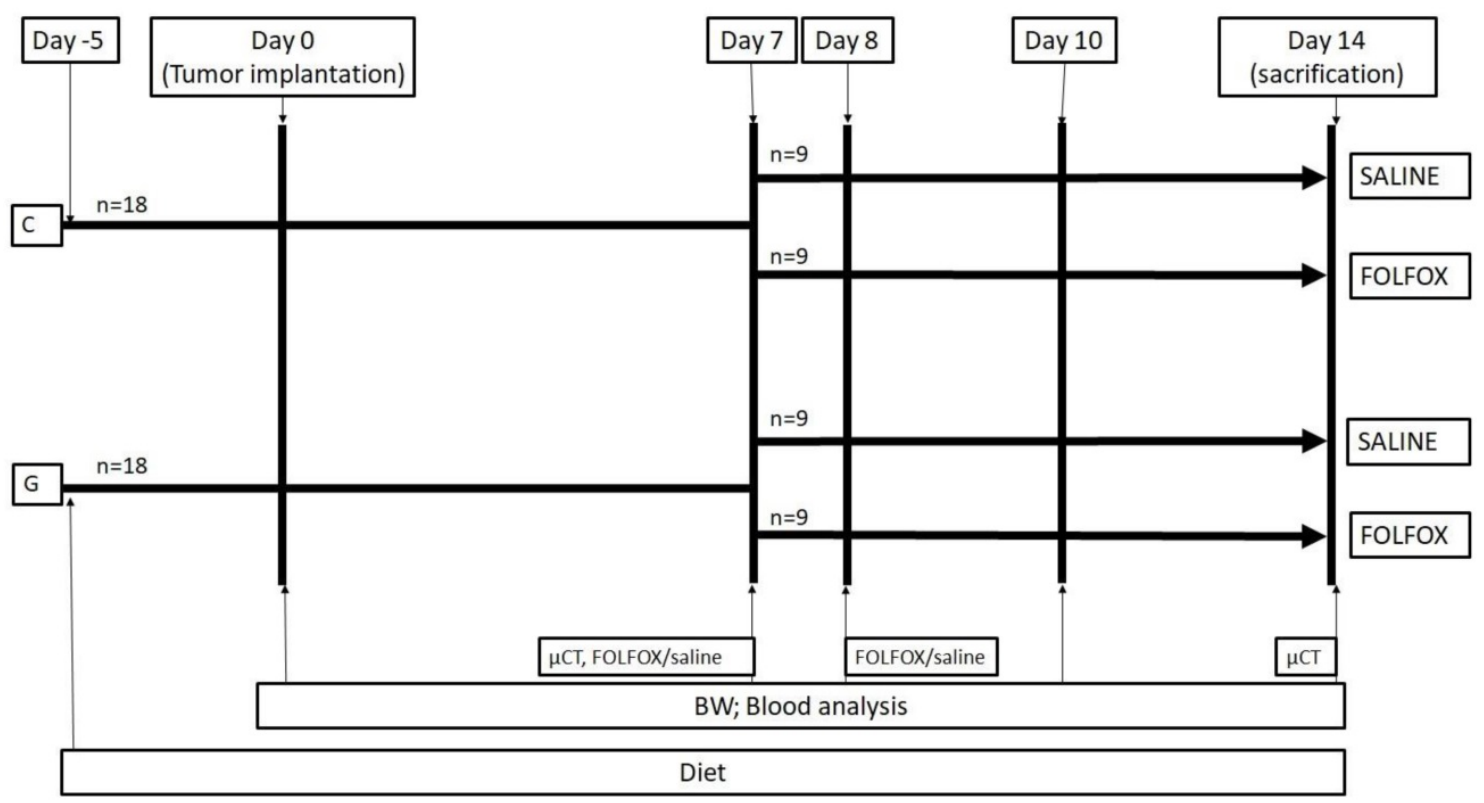

Figure 1. Experimental in vivo setting. Animals were fed for 5 days before experiments. At day 0 tumor was implanted into the liver and volume, MVD, Ki67, CT and blood work was done according to methods. Groups were split at day 7 and treated with or without FOLFOX. BW: body weight; FOLFOX: calcium folinate, oxaliplatin, and 5-Fluorouracil; G: 5\% glycine enriched diet; C: casein diet (controls). 
Immunohistochemistry. Tissue was taken at day 14 when all animals were sacrificed. For immunohistochemistry $3 \mu \mathrm{m}$ paraffin-embedded sections including healthy liver and tumor areas were prepared according to standard protocols. To evaluate tumor proliferation index the proliferation marker Ki67 (18) was used. Anti-Ki67 antibody (Thermo Fisher Scientific, Waltham, MA, USA; dilution 1:200, rabbit IgG Clone SP6) was used in combination with the UltraVision LP Detection System HRP Polymer (Thermo Fisher Scientific, Waltham, MA, USA) and DAB chromogen (Dako, Via Real Carpinteria, CA, USA). For positive control, rat intestinal tissue was used. For negative control, primary antibodies were omitted.

Microvascular density (MVD) in tumors was evaluated by staining against the endothelial cell marker CD31 (19). The anti-CD31 antibody (Abcam, Cambridge, UK; dilution 1:2000) was used in combination with the rabbit on rodent HRP-Polymer Detection System (Biocare Medical, CA, USA) and DAB chromogen (Dako, Via Real Carpinteria, CA, USA) exactly as described by the manufacturer. Hematoxyline was used for counterstaining for both staining procedures. For positive control, rat heart tissue was used. For negative control, primary antibodies were omitted. After staining all slides were scanned and images were viewed using the Aperio ImageScope ver.12.3.2.8013 software (Leica Biosystems Imaging, Wetzlar, Germany).

Quantification of Ki67 and CD31 staining was performed in 5 ( 3 peripheral and 2 central) randomly selected tumor areas from each slide. Snapshots at 100x magnification were taken and analyzed using ImageJ software (U. S. National Institutes of Health, Bethesda, Maryland, USA). For Ki67 analysis, IHC and classic watershed plugins were used. The proliferation index was counted as the stained nucleus ratio to a total number of nuclei.

Quantification of CD31 staining was performed using ImageJ software (U.S. National Institutes of Health, Bethesda, Maryland, USA). Single endothelial cells or cluster of cells positive for CD31 were considered as a vessel. After analysis MVD was compared between the different treatment groups.

Statistical analysis. Statistical analysis was performed using SPSS v.20.0 (SPSS Inc., Chicago, Illinois, USA). Data is presented as median and quartiles (Q1, Q3) unless stated differently. Differences between groups were analyzed using non-parametric tests - Mann Whitney $U$ test or Kruskal-Wallis test. For related sample analysis, the Wilcoxon-Signed rank test was used. All statistical tests were 2 -sided. P-values $<0.05$ were considered statistically significant.

\section{Results}

\section{In vitro data}

While glycine had no effect on CC531 cell viability in any concentration tested (Fig. 2a) both 5-FU (Fig. 2b) and OX (Fig. 2c) were cytotoxic to CC531. Glycine combined with 5-FU (Fig. 2b) and OX (Fig. 2c) did not have additional effects on cell viability.

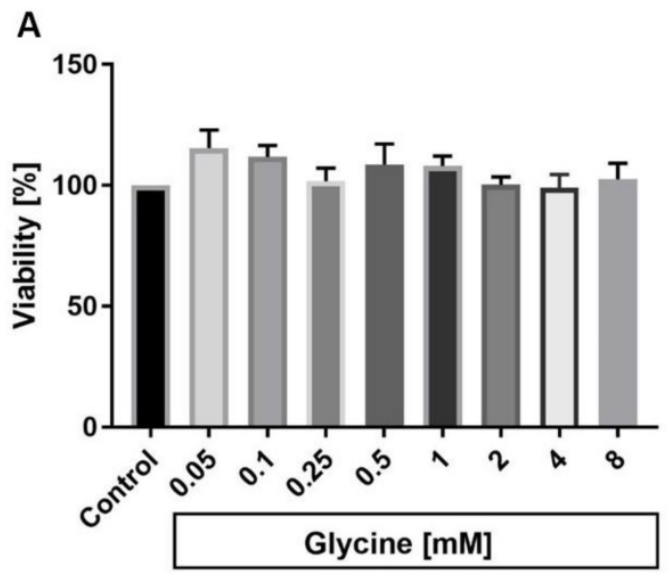

B

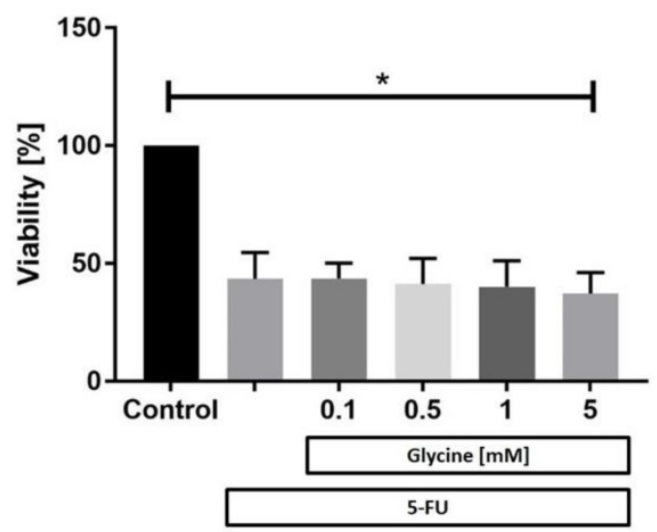

C

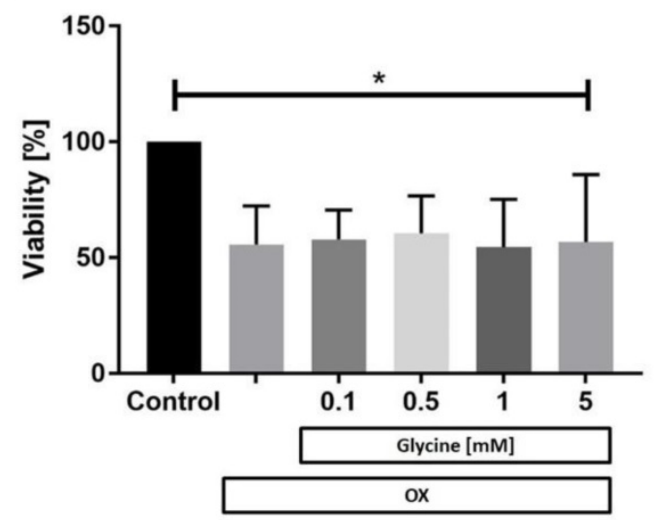

Figure 2. Cell viability in vitro. In vitro $C$ C531 viability was assessed after incubation with (a) various concentrations of glycine, (b) after incubation with 5-fluorouracil (5-FU) and (c) oxaliplatin (OX) at IC50 concentration combined with various concentrations of glycine. MTT assay was performed as described in methods. Results are presented as a mean \pm SD. * for significance with $p<0.05$. 


\section{Animal experiments}

General health conditions and body weight. FOLFOX did not have any effect on water consumption (data not shown) while food intake dramatically decreased from $12 \mathrm{~g}(9 ; 13.5)$ to $<1 \mathrm{~g} /$ rat per $24 \mathrm{~h}(\mathrm{p}<0.05)$. The effect was seen regardless of diet type and remained for $48 \mathrm{~h}$ with normal food consumption starting at day 9 (Table 1 ).

Table 1. Median body weight of the study groups at different time points of the study.

\begin{tabular}{lllll}
\hline Group & Day $0[\mathrm{~g}]$ & Day $7[\mathrm{~g}]$ & Day $10[\mathrm{~g}]$ & Day $14[\mathrm{~g}]$ \\
\hline Caseine & $220(200 ; 228)$ & $237(218 ; 246)$ & $247(225 ; 251)$ & $252(231 ; 257)$ \\
Glycine & $206(197 ; 212)$ & $219(213 ; 234)$ & $232(220 ; 242)$ & $236(225 ; 250)$ \\
$\begin{array}{l}\text { Caseine + } \\
\text { FOLFOX }\end{array}$ & $204(200 ; 218)$ & $227(223 ; 235)$ & $195(188 ; 198)$ & $173(168 ; 178)$ \\
$\begin{array}{l}\text { Glycine + } \\
\text { FOLFOX }\end{array}$ & $208(183 ; 215)$ & $220(197 ; 234)$ & $190(165 ; 200)$ & $169(150 ; 182)$ \\
\hline
\end{tabular}

The body weight (BW) of all animals was increasing from day 0 to day 7 . With FOLFOX the BW significantly decreased independently from diet to 83 $(81 ; 84) \%$ and $82(81 ; 94) \%$ in caseine+FOLFOX and glycine+FOLFOX subgroups, respectively (Tab. 1).

Hemoglobin and blood cell count. Hemoglobin (HGB) and red blood cell (RBC) levels remained stable through the whole study period without significant differences between the groups (data not shown). FOLFOX induced a significant decrease in white blood cells (WBC) which resulted in severe leukopenia. WBC levels in animals who received one cycle of FOLFOX decreased from $11.5 \times 10^{9} / 1$ (8.5; 12.9) prior to CTx to $0.5 \times 10^{9} / 1(0.37 ; 0.7)$ at the end of the experiment $(\mathrm{p}=0.001)$ (Fig. 3). Diet did not have an effect on WBC in FOLFOX treated rats at any time point (Fig. 3).
Glycine concentration. A glycine enriched 5 days diet increased the median plasma glycine concentration as compared with casein-fed rats by 8.7-fold (178 (152; 215) vs. 1549 (1332; 1819) $\mu \mathrm{mol} / \mathrm{l}$, $\mathrm{p}=0.001$ ) (Fig. 4). The presence of tumor did have no effect on glycine levels (Fig. 4). FOLFOX dramatically reduced the increase to only 1.4 -fold after glycine intake as compared with the corresponding casein group $(\mathrm{p}=0.001)$.

Liver and tumor volume. $\mu \mathrm{CT}$ liver scan on day 7 did not reveal any differences in liver and tumor volume between casein and glycine fed rats with 8913 $(7468 ; 10771) \mathrm{mm}^{3}$ vs $8045(5965 ; 8573) \mathrm{mm}^{3}$ and 67 $(25 ; 151) \mathrm{mm}^{3}$ vs $26(15 ; 77) \mathrm{mm}^{3}$. Further FOLFOX did not have an effect on liver volume with 7456 (6613; $8573) \mathrm{mm}^{3}$ compared with $8434(7841 ; 9401) \mathrm{mm}^{3}$ in corresponding saline controls on day 14 and dietary glycine did not change liver volume as compared with corresponding casein controls $(8403(7504 ; 10013)$ $\mathrm{mm}^{3}$ vs $\left.7814(6828 ; 8573) \mathrm{mm}^{3}\right)$.

Glycine $\pm F O L F O X$ reduced tumor growth from $292(193 ; 671) \mathrm{mm}^{3}$ and $271(140 ; 443) \mathrm{mm}^{3}$ to $123(193$; $671) \mathrm{mm}^{3}$ and $98(19 ; 165) \mathrm{mm}^{3}$, respectively $(\mathrm{p}<0.05)$ (Fig. 5). Moreover, tumors after glycine combined with FOLFOX were even smaller; however, differences failed to be significant (Fig. 5).

Proliferation index. Glycine did not change tumor proliferation with an index of $3.6(0.68 ; 7.99) \%$ compared with $4.06(1.97 ; 7) \%$ in the corresponding controls (Fig. 6). Further, FOLFOX did not decrease proliferation significantly from $4.06(1.97 ; 7) \%$ to 2.3 $(0.89 ; 3.44) \%$ (Fig. 6). Glycine combined with FOLFOX further decreased the tumor proliferation by $62 \%(0.89$ $(0.77 ; 1.8) \%)$; however, differences failed to be significant (Fig. 6).

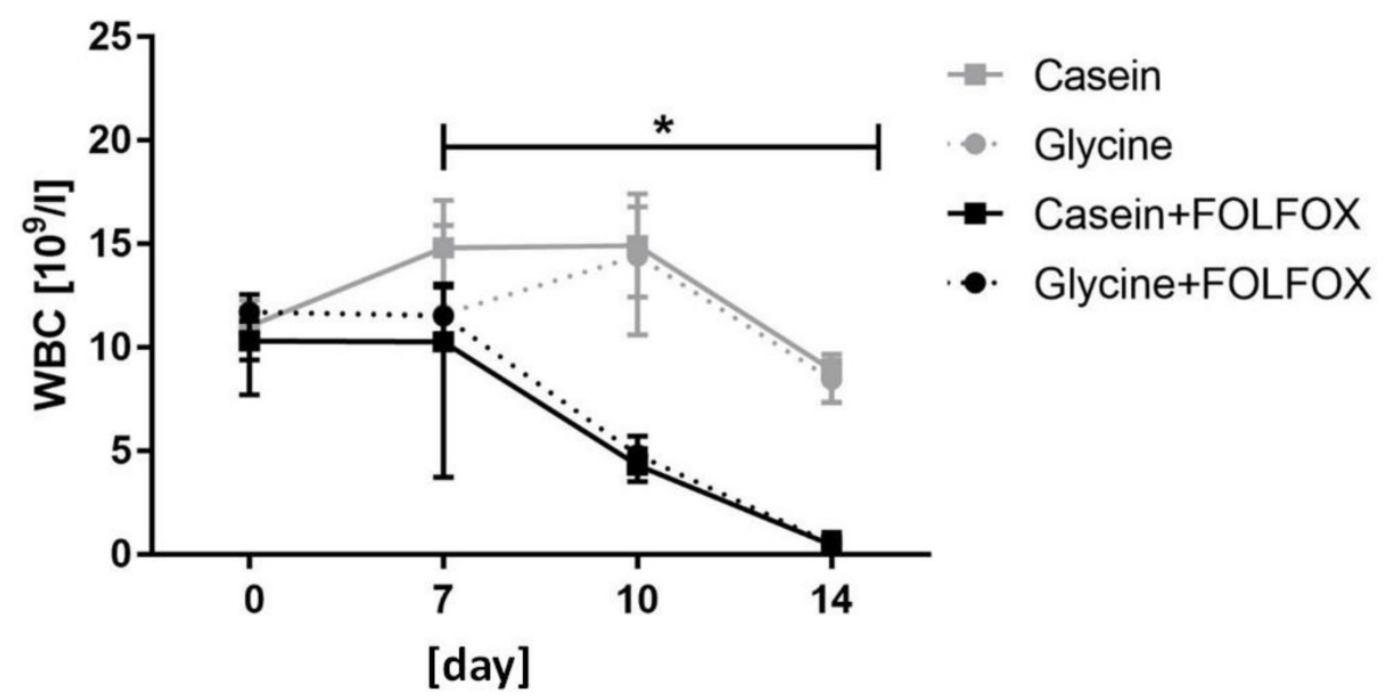

Figure 3. White blood cell count. Median WBC level significantly decreased in groups who received FOLFOX irrespective of diet. * for significance with $p<0.05$. 


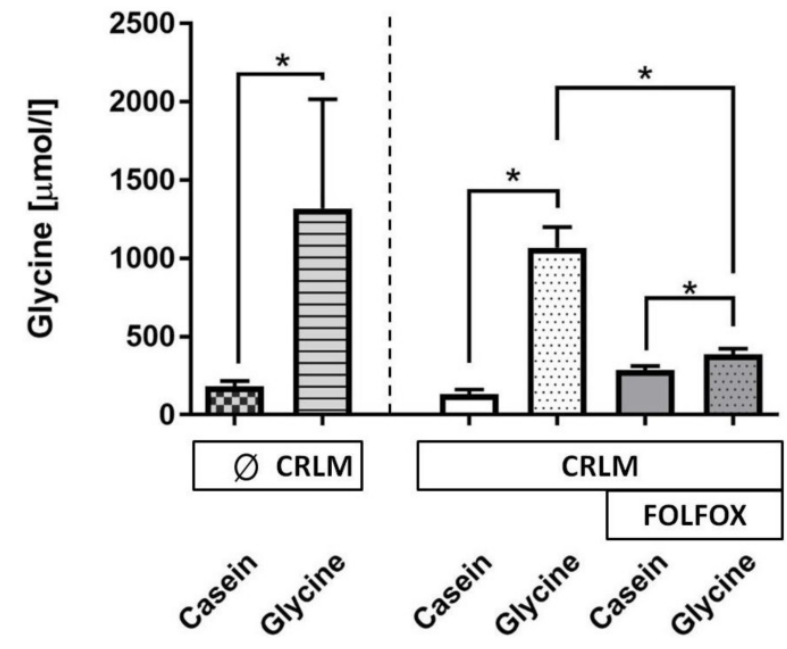

Figure 4. Glycine concentration $(\mu \mathrm{mol} / \mathrm{l})$ in plasma at the end of the experiment. Plasma glycine concentration was significantly increased in all groups treated with dietary glycine, but the level of the increase was significantly diminished with FOLFOX.* for significance with $\mathrm{p}<0.05$; CRLM - colorectal cancer liver metastasis.

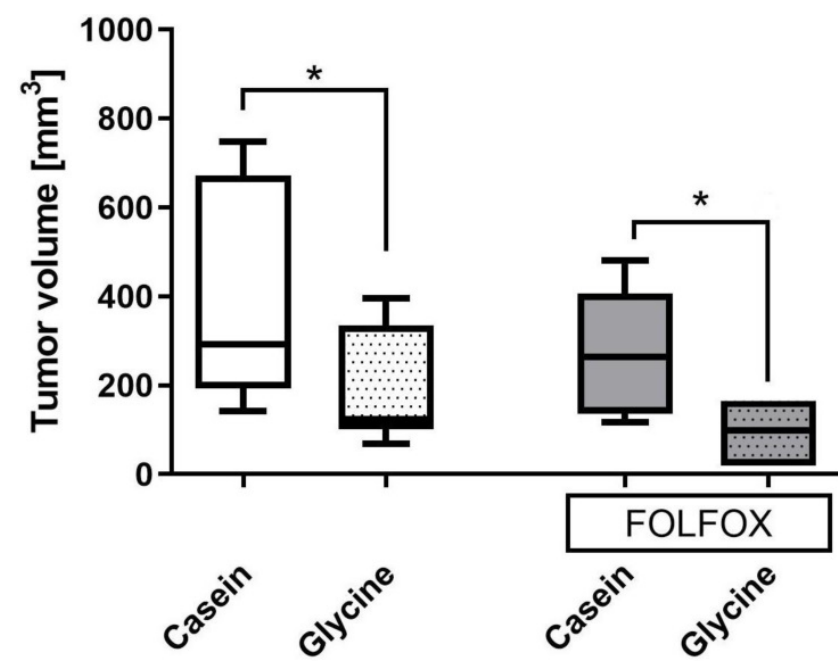

Figure 5. Tumor volume on the last day of the experiment in different treatment groups. Tumor volume calculated as described in methods. * for significance with $\mathrm{p}<0.05$.

Tumor vascularization. Glycine alone and combined with FOLFOX significantly decreased MVD from 53 and 46 to 16 and 23 MVD per field, respectively. CTx alone has no effect on MVD (Fig. 7).

\section{Discussion}

In this study, the anti-tumorigenic effect of dietary glycine was investigated in a combined in vivo model of CTx and CRLM for the first time. The results clearly show that dietary glycine significantly reduces the growth of CRLM. Rose et al. (14) reported, that glycine inhibits the growth of larger tumors to a greater extent than smaller tumors. The authors hypothesized that glycine prevents tumor growth by inhibiting tumor neo-vascularization. However, there was no evidence supporting this hypothesis and the speculations were mainly based on previous knowledge originating from a study, showing that dietary glycine inhibits subcutaneous melanoma tumor vascularization (13). Bruns et al. investigated the effect of glycine on endothelial cell (HUVEC) growth and angiogenesis and showed, that glycine has no direct impact on proliferation and viability of endothelial cells, but is capable of blunting VEGF stimulated HUVEC growth, migration and angiogenesis (9). Moreover, the authors demonstrated that HUVEC cells express a1 and a 2 subunits of the glycine receptor. Involvement of this glycine receptor to the inhibition process of angiogenesis was confirmed when the effect of glycine was neutralized after addition strychnine which has a greater affinity to the glycine receptor as compared with glycine (22).

Indeed, the glycine effect shown here was pronounced in a later tumor stage defined by the size of the tumor at a stage when tumors need vascularization for their further growth (Fig. 5). To date there was only indirect evidence for anti-angiogenic properties of glycine in vitro (9); however, data of this study clearly demonstrates that glycine reduces MVD in CRLM tumors (Fig. 7). Angiogenesis is crucial for tumor growth since the tumor exceeds a certain size where diffusion is a sufficient way to supply oxygen, nutrition and to eliminate metabolic waste $(21,22)$. Moreover, tumor vascularization is not only important for tumor growth but also predicts the outcome of the disease. A recent meta-analysis showed, that MVD measured by anti-CD31 staining in CRC tumors is inversely related to patients' survival (23). Taken together the results of this study showed, that glycine inhibits CRLM growth by decreasing microvascularization of the tumor.

In contrast, there is a controversial discussion on a possible direct pro-tumorigenic effect of glycine. A metabolite profiling study identified a key role of glycine in rapid cancer cell proliferation and suggested glycine metabolism as a target for therapeutic benefit (16). Further, a recent study showed that restriction of dietary serine and glycine can reduce CRC tumor growth in a mouse model (24). However, Labuschagne et al. widely investigated the role of serine and glycine in cancer cell metabolism and proliferation at the cellular level and demonstrated that restriction of exogenous glycine or depletion of the glycine cleavage system did not impede cancer cell proliferation (25). Indeed, this study has not shown increased CC531 viability even after cells were treated with high glycine doses of up to $8 \mathrm{mM}$ (Fig. 2). Moreover, here glycine did not have negative effects on the anti-tumorigenic properties of CTx in vitro. Further, glycine was nicely demonstrated to not increase the proliferation of tumor cells in vivo 
(Fig. 6). Ki67 to index proliferation is one of the most important immunocytochemical markers for cell proliferation (26) since its expression depends on the cell cycle and it is expressed constitutively except for cell is in resting (G0) phase (27). In this study, no negative effect on blood cells could be found at any time point (Fig. 3). While glycine had no impact on Ki67, one cycle of FOLFOX decreased cell proliferation; however, values did not reach significance. This is strong evidence for glycine being synergistic with FOLFOX working through different pathways. Both 5-FU and OX are directly cytotoxic to cells while glycine decreases tumor volume by the reduction of its vascularization. Clinical trials have already shown the benefit of anti-angiogenic agents given together with a fluorouracil- or oxaliplatin-based chemotherapy (27-32). Indeed, glycine data shown here dramatically increased the anti-tumorigenic effect by reducing the tumor volume especially if combined with on cycle of FOLFOX. More cycles of FOLFOX were not possible due to its side effects, i.e. severe leukopenia, significant weight loss and poor general condition of the animals.
The currently approved anti-angiogenic agents, such as Bevacizumab, Zivaflibercept, Ramucirumab, Regorafenib carry the great risk of various severe adverse events such as hypertension, proteinuria, arterial thrombosis, bleeding, neutropenia or various skin reactions $(33,34)$. In contrast, orally or intravenously applied glycine was proven in large clinical trials to have no relevant toxicity (9-12). Moreover, a study performed by Mikalauskas et al. demonstrated the hepatoprotective effect of non-toxic glycine against FOLFOX- or FOLFIRI-induced liver injury (15). The combination of glycine and currently used anti-VEGF antibodies were never tested in vitro or in vivo. Although, these two-different anti-angiogenic agents, work through different mechanisms. The anti-VEGF antibodies directly bind to VEFG extracellularly and prevent interaction with VEGF receptors (35), while glycine blunts VEGF stimulated endothelial cells growth, migration, and angiogenesis via mechanisms involving glycine receptor (9). Therefore, the risk of negative interaction between these two agents seems low.
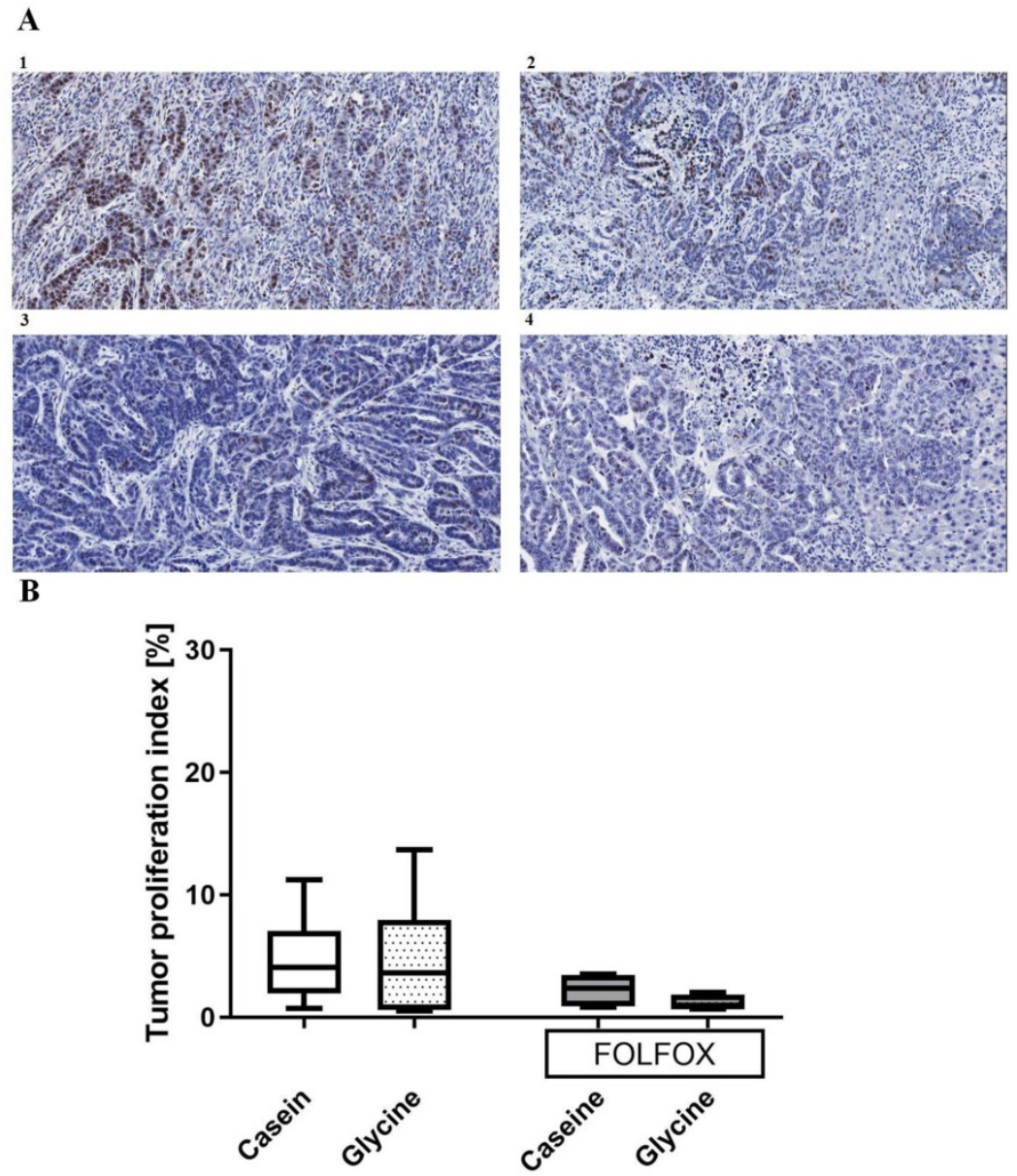

Figure 6. Tumor proliferation index. A; Representative pictures of tumors stained with anti-Ki67 antibody in (1) casein, (2) glycine, (3) FOLFOX+casein and (4) FOLFOX+glycine. B; comparison of proliferation index between the groups. Both groups treated with FOLFOX showed a tendency $(p<0.1)$ to have a lower tumor proliferation index, however, the differences were not significant. 

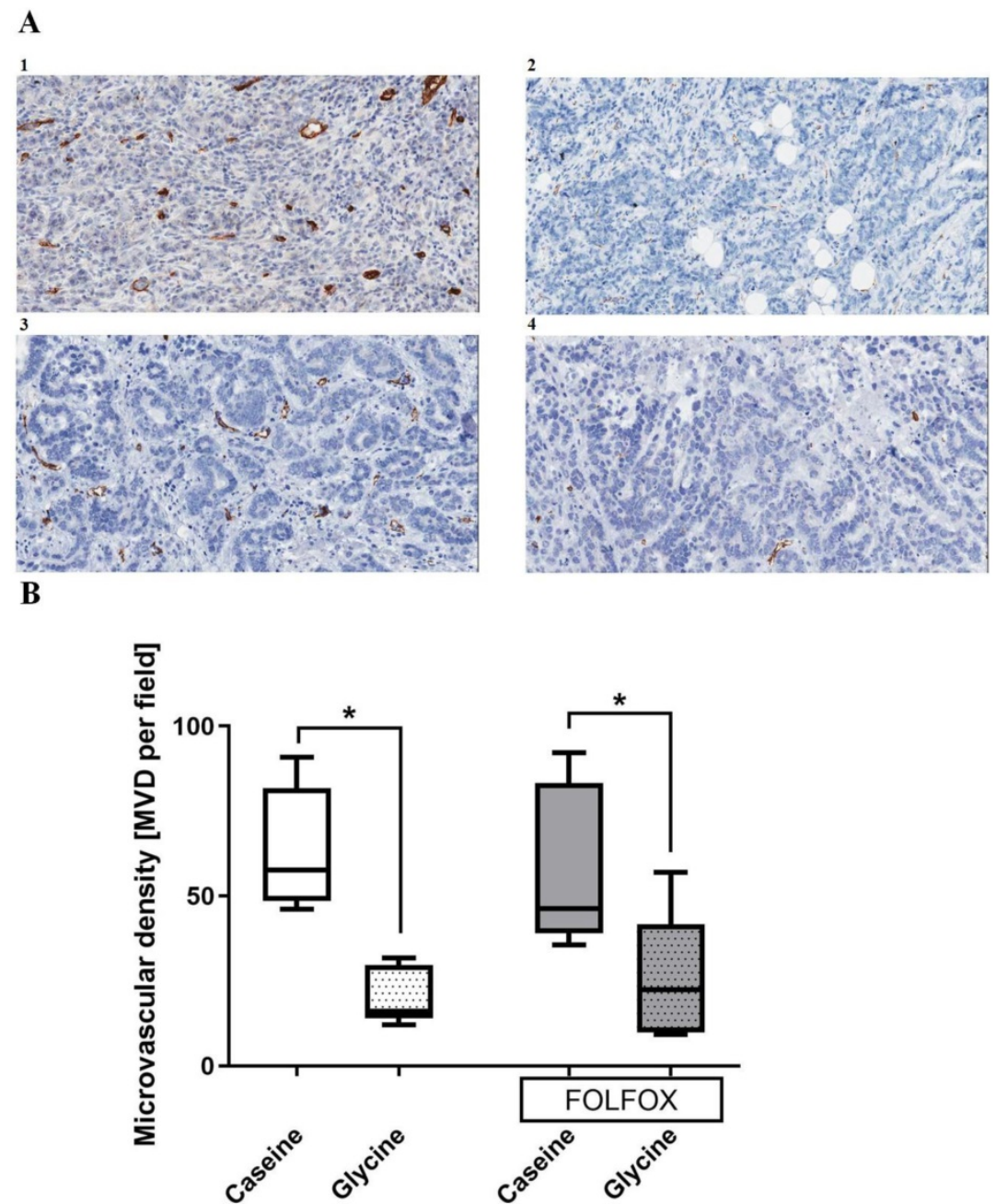

Figure 7. Tumor microvascular density (MVD) in different treatment groups. A; representative pictures of tumors stained with anti-CD31 antibody in (1) casein, (2) glycine, (3) FOLFOX+casein and (4) FOLFOX+glycine. B; comparison of MVD between groups. Glycine significantly reduced the MVD irrespective of FOLFOX; * for significance with $p<0.05$.

While glycine values were significantly lower with FOLFOX (Fig. 4) its effect on MVD was comparable (Fig. 7). How can the FOLFOX effect on glycine levels be explained? Firstly, the total dietary uptake decreased significantly with FOLFOX. Secondly, there could be insufficient drug uptake (36) related to mal-absorption by the intestinal mucositis after FOLFOX $(37,38)$.

\section{Conclusion}

Data of this study clearly demonstrates that glycine nicely decreases tumor volume. Underlying mechanisms most likely include a significantly decreased MVD in tumors. Glycine has additive indirect anti-tumor effects if given together with FOLFOX. While anti-angiogenic properties of glycine are not toxic in contrast to the clinically established drugs clinical trials are warranted to assess its value in the human setting.

\section{Abbreviations}

5-FU: 5-fluorouracil; CBC: complete blood count; CC531: rat colorectal cancer cell line; CRC: colorectal cancer; CRLM: colorectal liver metastases; CTx: chemotherapy; FOLFOX: chemotherapy scheme of 5-fluorouracil, oxaliplatin, leucovorin; HGB: hemoglobin; IC50: half maximal inhibitory concentration; MTT: 3-(4,5-dimethylthiazol-2-yl)5diphenyltetrazolium bromide; MVD: tumor microvascular density; OX: oxaliplatin; PBS: phosphate-buffered saline; RBC: red blood cell; VEGF: vascular endothelial growth factor; WBC: white blood cells.

\section{Competing Interests}

The authors have declared that no competing interest exists. 


\section{References}

1. Torre LA, Bray F, Siegel RL, Ferlay J, Lortet-Tieulent J, Jemal A. Global cancer statistics, 2012. CA Cancer J Clin. 2015 Mar;65(2):87-108.

2. Sperling J, Brandhorst D, Schäfer T, Ziemann C, Benz-Weißer A, Scheuer C, et al. Liver-directed chemotherapy of cetuximab and bevacizumab in combination with oxaliplatin is more effective to inhibit tumor growth of CC531 colorectal rat liver metastases than systemic chemotherapy. Clin Exp Metastasis. 2013 Apr;30(4):447-55.

3. Arnold M, Sierra MS, Laversanne M, Soerjomataram I, Jemal A, Bray F. Global patterns and trends in colorectal cancer incidence and mortality. Gut. 2017 Apr;66(4):683-91.

4. Zervoudakis A, Boucher T, Kemeny NE. Treatment Options in Colorectal Liver Metastases: Hepatic Arterial Infusion. Visc Med. 2017;33(1):47-53.

5. Sanoff HK, Sargent DJ, Campbell ME, Morton RF, Fuchs CS, Ramanathan RK, et al. Five-Year Data and Prognostic Factor Analysis of Oxaliplatin and Irinotecan Combinations for Advanced Colorectal Cancer: N9741. J Clin Oncol. 2008 Dec 10;26(35):5721-7.

6. Toden S, Okugawa Y, Jascur T, Wodarz D, Komarova NL, Buhrmann C, et al. Curcumin mediates chemosensitization to 5-fluorouracil through miRNA-induced suppression of epithelial-to-mesenchymal transition in chemoresistant colorectal cancer. Carcinogenesis. 2015 Mar;36(3):355.

7. Fang L, Yang Z, Zhou J, Tung J-Y, Hsiao C-D, Wang L, et al. Circadian clock gene CRY2 degradation is involved in chemoresistance of colorectal cancer. Mol Cancer Ther. 2015 Jun;14(6):1476.

8. Vogel A, Hofheinz RD, Kubicka S, Arnold D. Treatment decisions in metastatic colorectal cancer - Beyond first and second line combination therapies. Cancer Treat Rev. 2017 Sep 1;59:54-60.

9. Bruns H, Kazanavicius D, Schultze D, Saeedi MA, Yamanaka K, Strupas K, et al. Glycine inhibits angiogenesis in colorectal cancer: role of endothelial cells. Amino Acids. 2016 Nov;48(11):2549-58

10. Evins AE, Fitzgerald SM, Wine L, Rosselli R, Goff DC. Placebo-Controlled Trial of Glycine Added to Clozapine in Schizophrenia. Am J Psychiatry. 2000 May 1;157(5):826-8.

11. Gannon MC, Nuttall JA, Nuttall FQ. The metabolic response to ingested glycine. Am J Clin Nutr. 2002 Dec 1;76(6):1302-7.

12. Kaufman MJ, Prescot AP, Ongur D, Evins AE, Barros TL, Medeiros CL, et al. Oral glycine administration increases brain glycine/creatine ratios in men: a proton magnetic resonance spectroscopy study. Psychiatry Res. 2009 Aug 30;173(2):143-9.

13. Rose ML, Madren J, Bunzendahl H, Thurman RG. Dietary glycine inhibits the growth of B16 melanoma tumors in mice. Carcinogenesis. 1999 May;20(5):793-8.

14. Rose ML, Cattley RC, Dunn C, Wong V, Li X, Thurman RG. Dietary glycine prevents the development of liver tumors caused by the peroxisome proliferator WY-14,643. Carcinogenesis. 1999 Nov;20(11):2075-81.

15. Mikalauskas S, Mikalauskiene L, Bruns H, Nickkholgh A, Hoffmann K, Longerich $\mathrm{T}$, et al. Dietary glycine protects from chemotherapy-induced hepatotoxicity. Amino Acids. 2011 Apr;40(4):1139-50.

16. Jain M, Nilsson R, Sharma S, Madhusudhan N, Kitami T, Souza AL, et al. Metabolite Profiling Identifies a Key Role for Glycine in Rapid Cancer Cell Proliferation. Science. 2012 May 25;336(6084):1040-4.

17. Field KM, Kosmider S, Jefford M, Jennens R, Green M, Gibbs P. Chemotherapy Treatments for Metastatic Colorectal Cancer: Is Evidence-Based Medicine in Practice? J Oncol Pract. 2008 Nov 1;4(6):271-6.

18. Ma X, Wu Y, Zhang T, Song H, Jv H, Guo W, et al. Ki67 Proliferation Index as a Histopathological Predictive and Prognostic Parameter of Oral Mucosal Melanoma in Patients without Distant Metastases. J Cancer. 2017 Oct 17;8(18):3828-37.

19. Lazaris A, Amri A, Petrillo SK, Zoroquiain P, Ibrahim N, Salman A, et al. Vascularization of colorectal carcinoma liver metastasis: insight into stratification of patients for anti-angiogenic therapies. J Pathol Clin Res 2018 Jul;4(3):184-192

20. Cabrera-Pastor A, Malaguarnera M, Taoro-Gonzalez L, Llansola M, Felipo V. Extracellular cGMP Modulates Learning Biphasically by Modulating Glycine Receptors, CaMKII and Glutamate-Nitric Oxide-cGMP Pathway. Sci Rep. 2016 Sep 16;6:33124.

21. Takahashi Y, Ellis LM, Mai M. The angiogenic switch of human colon cancer occurs simultaneous to initiation of invasion. Oncol Rep. 2003 Jan 1;10(1):9-13.

22. Nagy JA, Brown LF, Senger DR, Lanir N, Van De Water L, Dvorak AM, et al. Pathogenesis of tumor stroma generation: a critical role for leaky blood vessels and fibrin deposition. Biochim Biophys Acta BBA - Rev Cancer. 1989 Feb;948(3):305-26

23. Guetz GD, Uzzan B, Nicolas P, Cucherat M, Morere J-F, Benamouzig R, et al. Microvessel density and VEGF expression are prognostic factors in colorectal cancer. Meta-analysis of the literature. Br J Cancer. 2006 Jun 19;94(12):1823.

24. Maddocks ODK, Athineos D, Cheung EC, Lee P, Zhang T, Broek NJF van den, et al. Modulating the therapeutic response of tumours to dietary serine and glycine starvation. Nature. 2017 Apr;544(7650):372.

25. Labuschagne CF, van den Broek NJF, Mackay GM, Vousden KH, Maddocks ODK. Serine, but not glycine, supports one-carbon metabolism and proliferation of cancer cells. Cell Rep. 2014 May 22;7(4):1248-58.

26. Zhao W-Y, Xu J, Wang M, Zhang Z-Z, Tu L, Wang C-J, et al. Prognostic value of Ki67 index in gastrointestinal stromal tumors. Int J Clin Exp Pathol. 2014 Apr 15;7(5):2298-304.
27. Kontzoglou K, Palla V, Karaolanis G, Karaiskos I, Alexiou I, Pateras I, et al. Correlation between Ki67 and Breast Cancer Prognosis. Oncology. 2013;84(4):219-25.

28. Hurwitz H, Fehrenbacher L, Novotny W, Cartwright T, Hainsworth J, Heim $\mathrm{W}$, et al. Bevacizumab plus irinotecan, fluorouracil, and leucovorin for metastatic colorectal cancer. N Engl J Med. 2004 Jun 3;350(23):2335-42.

29. Saltz LB, Clarke S, Díaz-Rubio E, Scheithauer W, Figer A, Wong R, et al. Bevacizumab in combination with oxaliplatin-based chemotherapy as first-line therapy in metastatic colorectal cancer: a randomized phase III study. J Clin Oncol Off J Am Soc Clin Oncol. 2008 Apr 20;26(12):2013-9.

30. Tebbutt NC, Wilson K, Gebski VJ, Cummins MM, Zannino D, van Hazel GA, et al. Capecitabine, bevacizumab, and mitomycin in first-line treatment of metastatic colorectal cancer: results of the Australasian Gastrointestinal Trials Group Randomized Phase III MAX Study. J Clin Oncol Off J Am Soc Clin Oncol. 2010 Jul 1;28(19):3191-8.

31. Giantonio BJ, Catalano PJ, Meropol NJ, O'Dwyer PJ, Mitchell EP, Alberts SR, et al. Bevacizumab in combination with oxaliplatin, fluorouracil, and leucovorin (FOLFOX4) for previously treated metastatic colorectal cancer: results from the Eastern Cooperative Oncology Group Study E3200. J Clin Oncol Off J Am Soc Clin Oncol. 2007 Apr 20;25(12):1539-44.

32. Masi G, Salvatore L, Boni L, Loupakis F, Cremolini C, Fornaro L, et al. Continuation or reintroduction of bevacizumab beyond progression to first-line therapy in metastatic colorectal cancer: final results of the randomized BEBYP trial. Ann Oncol Off J Eur Soc Med Oncol. 2015 Apr;26(4):724-30.

33. Elice F, Rodeghiero F. Side effects of anti-angiogenic drugs. Thromb Res. 2012 Apr;129 Suppl 1:S50-53.

34. Riechelmann R, Grothey A. Antiangiogenic therapy for refractory colorectal cancer: current options and future strategies. Ther Adv Med Oncol. 2017 Feb;9(2):106-26.

35. Niu G, Chen X. Vascular Endothelial Growth Factor as an Anti-angiogenic Target for Cancer Therapy. Curr Drug Targets. 2010 Aug;11(8):1000-17.

36. Cobo-Dols M, Pérez-Miranda E, Gil-Calle S, Alés-Díaz I, Villar-Chamorro E, Montesa-Pino A, et al. Changes in the serum amino acids concentrations after first cycle as a factor predictive of tumor response to chemotherapy. Oncol Barc. 2005 Jul;28(7):21-8.

37. Renck D, Santos AA, Machado P, Petersen GO, Lopes TG, Santos DS, et al. Human uridine phosphorylase-1 inhibitors: a new approach to ameliorate 5-fluorouracil-induced intestinal mucositis. Invest New Drugs. 2014 Dec;32(6):1301-7.

38. Abdel-Rahman O, ElHalawani H, Essam-Eldin S. S-1-based regimens and the risk of oral and gastrointestinal mucosal injury: a meta-analysis with comparison to other fluoropyrimidines. Expert Opin Drug Saf. 2016 Jan;15(1):5-20 